plished by instruments. 5. In timid persons and children, in whom opcrative procedures meet with great obstacles, and often can only be exccuted under anæsthetics.-Med. Times and Gaz., Nov. 6, 1880.

\title{
MIDWIFERY AND GYNÆCOLOGY.
}

\section{The Management of the Third Stage of Labour.}

Dr. Max Runge, in a communication to the Obstetrical Soeiety of Bcrlin, criticizes the current teaching regarding the management of the third stage of labour. He takes as the espccial text of his animadversions the directions given by Fritsch, which are to the effect that immediately after the birth of the child the uterus is to be seized by the hand on the abdomcn, and the placenta pressed out. Dr. Runge states that for a long time he faithfully carried out this method; and so did others in Professor Gusserow's clinique. The objection to it is, that the squeezing out of the placenta is begun before that organ has become completely separated; consequently, when the placenta has been expellcd, of ten a bit of the membranes may yet be attached to the utcrus, and be left behind after the placenta has been taken away. While this teaching was carried out it was quite a common thing for a pair of forceps to be needed to remove these retained pieces of membrane; and secondary post-partum hemorrhage becamc extraordinarily frequent. He refers to a former communication of his own, in which, treating of post-partum hemorrhage, he expressed his surprise that within a short time he had had many cases of this complication. Then he supposed this frequency was fortuitous. Now he knew the reason, which was his undue haste in pressing out the placenta. Midwives are now instructed, after the birtl of the child (and having, of course seen that the uterus is sufficiently contracted upon the placenta to prevent hemorrhage), to wash and dress the infunt before proceeding to press out the placenta. The separation of the placenta and membranes, Dr. Runge holds, is not complete until, upon an averagc, about a quarter of an hour after the birth of the child; and therefore about this length of time should be allowed to elapse before the placenta is pressed out. Since instructions based upon this principle have been given to the students and midwives of the Strasburg Obstetric Clinique, post-partum hemorrlage has become of very infrequent occurrence.Med. Times and Gaz., Nov. 20, 1880.

\section{Incomplete Internal Spontancous Rupture of the Uterus.}

Dr. Oтto AlBents, of Berlin, publishes a case (Berliner Klinische Wochenschrift, No. 45, 1880) in which the above diagnosis was made. He saw the patient first after she had been in labour about seven hours. The pelvis was slightly contracted (conjugate between ten and cleven centimetres); the pains vigorous and frequent, but not prorlucing much effect. He adviscd cxpectant treatment. Two hours later he was again called, and learned that the pains had suddenly ceaser, and that the head had slightly receded. He found the belly very tender, the contour of the utcrus indistinct and its consistence soft; and the fortal limbs felt with extraordinary distinctness. There were no pains ; the cervix uteri hung soft and loose in the vagina, and the head was high and movable above the pelvic brim. But the signs of deep collapse which usually accompany com- 
plete rupture of the uterus were here absent. Immediate delivery was decided upon. Podalic version was performed, and the delivery of the head accelerated by perforation. The placenta followed spontaneously; the uterus contracted well, and there was no post-partum hemorrhage. Ergot was given. In the course of the day a tumour became perceptible in the right side of the hypogastric region, of the size of the fist, smooth, and circumscribed; this was believed to be a sub-peritoneal hæmatoma. This at first increased, and extended down into the right labium majus. When by manipulation the uterus was made to contract, this tumour was also felt to get hard. Seven weeks after delivery the patient went out, well. On account of the pelvic contraction, she was advised, in the event of a subsequent pregnancy, to have labour brought on prematurely. She became pregnant again, but neglected the advice given. Labour came on at term, and complete rupture of the uterus took place, the anterior wall being torn across, so as to separate the body from the neck of the uterus. The author considers that this rent must have begun in the cicatrix of the one believed to have formerly taken place.-Med. Times and Gazette, Nov. 27, 1880.

\section{Porro Operation.}

Professor G. VeIT records (Zeitschr. f. Geb. u. Gynak., v. 2, 1880) a case of Porro's operation which ended fatally, although death was not due to the operation itself. Facial erysipelas supervened on the third day, the patient having done well up to that time, and death took place within twenty-four hours. A small abscess was found in each kidney. In this case the operative procedure was somewhat modified, in accordance with hints drawn from Freund's opcration of total cxtirpation of the utcrus and Schröder's method of closing the stump with sutures after removal of the organ for tumours. Moreover, the constriction of the cervix was only temporary, an Esmarch's bandage being used for the purpose. The constrictor was tightened beforc the uterus was opened, and yet the child cried at once on being extracted. The abdominal incision was about eighteen centimetres long, beginning three centimctres above the symphysis. The uterus, still intact, was easily drawn out through this opening. At the same time two coils of small intestine were extruded, but they were easily replaced, and, to prevent a repctition of this occurrence, the upper part of the abdominal wound was closed with sutures at once. An Esmarch's bandage was now thrown around the isthmus, six centimetres below the ovaries, the uterus was opcned, and the child was extracted. Only a very littlc venous blood issued from the uterine incision. Each broad ligament was now tied with two ligatures, the inner one reaching to the border of the isthmus, and the ligaments were then cut through. On the right side the uterine artery was seen to have been divided, and a special ligature was applied to it. The uterus was now severed by an incision near the isthmus. To close the stump, four deep sutures, including the whole thickness of the cervix, were applied, together with twelve superficial sutures. At this juncture, on removing the constrictor, a stream of blood from the left uterine artery welled up between the sutures. This was controllcd by passing a double ligature through the stump, onc thread of which was tied at the left-hand murgin of the stump and the other at its top. The abdominal wound was closed with thirty sutnres. The whole operation, with only one assistant, was over in three-quarters of an hour. The author suggests that, by rupturing the membranes at the beginning of the operation, the uterus may be lessened in size somewhat, so that it may be brought out through a comparatively short abdominal incision.-New York Medl. Journal, Oct. 1880. 